\title{
An Investigation of the Differences Between On-road and Wind-tunnel Noise
}

\author{
Peter O'Shea ${ }^{(1)}$, Simon Watkins ${ }^{(2)}$, Christian Peric ${ }^{(2)}$ and Bruce Sloss ${ }^{(2)}$ \\ (1) Dept. of Communication \& Electronic Engineering, Royal Melbourne Institute of Technology, \\ (2) Dept. of Mechanical Engineering, Royal Melbourne Institute of Technology, \\ GPO Box 2476V, Melbourne, Australia 3001
}

\begin{abstract}
The airflow around a moving car results in wind noise which is partially transmitted into the cabin. With the trend towards quieter cars, this wind-generated noise can dominate other noise sources. Standard methods of developing car geometries (often with the aim of minimising wind noise) are based on tests in smooth-flow wind tunnels, rather than by road trials. However, it has been noted that the sound of the wind is perceptually different in the two test environments. This paper sets out to characterise these acoustical differences. Higher-order spectral statistics and wavelet analysis have been utilised and significant variations in the two environments were found. Ultimately, it is hoped that such analyses can be used to predict on-road wind noise from smooth flow wind-tunnel data (utilising knowledge of the turbulent velocity fluctuations in the atmosphere).
\end{abstract}

\section{INTRODUCTION}

The reduction of car cabin noise forms a significant part of modern car development and mechanical noises (engine, transmission and tyre) are now low. The reduction of wind noise is thus receiving considerable attention, with some automotive manufacturers building aeroacoustic tunnels dedicated to the measurement of wind noise. (Such tunnels are characterised by quiet, low turbulence flow and hemianechoic test sections). However, the on-road wind conditions relative to the car vary continuously in time and space mainly because of fluctuations in the atmospheric wind; ${ }^{1}$ hence the wind noise in realistic on-road environments varies with time. This is not the case in wind tunnels. ${ }^{2}$ Since the characteristics of velocity fluctuations in atmospheric turbulence are well known, it is attractive to attempt to predict the wind noise that would occur under "real" on-road conditions utilising data obtained in wind tunnels.

\section{TEST PROCEDURES}

In order to document wind noise and wind conditions, a modern passenger car was fitted with an Aachen artificial head system in the front passenger seat and hot-wire anemometers were located upstream of the "A" pillar (to measure velocity fluctuations in the oncoming flow). Data presented in this paper were recorded on an instrumentation quality digital audio tape (DAT) and were sampled at either 24,000 Hz (for the data in Figs. 7 and 8) or 35,000 Hz (data for all other figures). Initial data analysis, performed at various sampling frequencies, showed no significant levels of sound energy were present above about $17,000 \mathrm{~Hz}$. A high pass filter which was built into the Aachen Head System, and which had a cut-off frequency of $22.4 \mathrm{~Hz}$, was used.

The test vehicle was used to acquire noise and velocity data on a road that was characterised by negligible roadside structures (vegetation, buildings etc.) and during times of no traffic. Thus the velocities relative to the moving vehicle were the vector sum of the atmospheric wind and vehicle road speed. Data presented here were recorded at a road speed of $120 \mathrm{~km} / \mathrm{h}$ with a direct headwind of approximately $20 \mathrm{~km} / \mathrm{h}$. Thus the relative velocity was close to $140 \mathrm{~km} / \mathrm{h}$ with negligible yaw angle.

Data were also recorded in the Monash/RMIT Vehicle Aeroacoustic Wind Tunnel. This tunnel has a background noise level that is $10 \mathrm{~dB}$ or more below the wind noise level of the test vehicle for the frequencies of interest. This is considered adequate for wind noise testing without corrections being needed. ${ }^{3}$ Tests were performed at a wind-tunnel speed of $140 \mathrm{~km} / \mathrm{h}$ and with the vehicle unyawed, thus closely replicating the relative air velocity experienced by the test vehicle on the road test. The wind tunnel had a longitudinal turbulence intensity of $1 \%$ and is a closed circuit, $3 / 4$ open jet, wind tunnel with a maximum velocity of $160 \mathrm{~km} / \mathrm{h}$. Acoustic wedges, to replicate a free acoustic field, are planned to be installed, but were not present for these tests. Details of the tunnel can be found in reference. ${ }^{4}$

\section{PROCESSING OF THE ACOUSTIC SIGNALS}

The acoustic spectra of both on-road and wind-tunnel tests were acoustically adjusted with A-weighting, averaged over time and compared, with a typical result being shown in Fig. 1. The averaging was performed on 1024 point FFTs which were overlapped by $50 \%$. It can be seen that the averaged sound spectra were only marginally different in the region above $400 \mathrm{~Hz}$, where wind noise dominates. ${ }^{5}$ However, amplitude fluctuations of very low frequency (approximately $1 \mathrm{~Hz}$ ) could clearly be heard in the on-road wind noise. As engine speed, load and road characteristics were constant, it was postulated that these fluctuations must result from wind gusts. These variations were not apparent in the averaged acoustic spectra, because the averaging procedure masks them out.

As suggested above, it has been hypothesised that there is a perceptual difference between wind-tunnel noise and on-road noise because of the time variations in the spectra of the on-road noise. That is, it is conjectured that the on-road data has statistical moments which vary with time much more 